\title{
Hypertrophic repair of articular cartilage in experimental osteoarthrosis
}

\author{
ERIC VIGNON, ${ }^{1}$ MONIQUE ARLOT,${ }^{2}$ DANIEL HARTMANN, ${ }^{3}$ \\ BERNARD MOYEN, ${ }^{4}$ AND G. VILLE ${ }^{3}$
}

From the ${ }^{1}$ Department of Rheumatology, Hôpital E. Herriot, Lyon; ${ }^{2}$ INSERM, Unité 234, Lyon; the ${ }^{3}$ Centre of Radioanalysis, Institut Pasteur, Lyon; and the ${ }^{4}$ Department of Orthopaedic Surgery, Lyon, France

SUMMARY Section of the anterior cruciate ligament has been performed in the knee of 11 mature dogs. The macroscopically normal cartilage from patella and femoral trochlea of animals killed from 2 to 32 weeks after operation was used for histological, histomorphometrical, and biochemical analysis. Previously undescribed degenerative lesions of the superficial matrix were observed, and there was evidence for secondary healing of these lesions. An early and progressive decrease in superficial cell density and a later progressive increase in cartilage thickness without any change in the cell density of the middle and deep cartilage layers was found. A slight increase in water content with no reduction in glycosaminoglycan content was observed. The results suggest that joint laxity results initially in superficial degenerative changes and later in hypertrophic regenerative changes due to cell proliferation and increased matrix synthesis. Hypertrophic remodelling of articular cartilage in response to abnormal mechanical stresses is postulated.

Experimental osteoarthritis offers a unique opportunity for the study of the initial events leading to cartilage destruction, which is the main characteristic of the disease. Various models of osteoarthritis can be used, ${ }^{1}$ but joint laxity induced by section of a cruciate ligament of the knee or meniscectomy is now considered the most valid method. ${ }^{2-6}$ Joint-laxityinduced osteoarthritis has been used for numerous biochemical and metabolic studies, and changes in water content and proteoglycan preceding the appearance of cartilage fibrillation have been demonstrated. ${ }^{45}$ In contrast the early histological changes are far less documented, and histomorphometric data are still lacking.

Here we report the results of a study of the qualitative and quantitative histological changes preceding cartilage fibrillation induced by section of the anterior cruciate ligament of the dog's knee. We restricted the study to the cartilage of the patella and femoral condyle, which is only slowly affected by fibrillation. Histological data were correlated with glycosaminoglycan and water content.

\section{Materials and methods}

Animals and surgical procedures. A total of $11 \mathrm{dogs}$ Accepted for publication 23 December 1981.

Correspondence to Dr E. Vignon, Pavillon E, Hôpital Edouard Herriot, Place d'Arsonval, 69374 Lyon Cedex 2, France. were used. They were of unselected breed and of both sexes, were 3 to 8 years old, and weighed 16 to $30 \mathrm{~kg}$. Absence of prior osteoarthritis was established by clinical examination and during surgical procedures. The dogs were anaesthetised with intravenous sodium pentothal. The anterior cruciate ligament in the right knee of each dog was exposed through a median parapatellar incision and transected with a scalpel, care being taken to avoid damage to the articular surfaces. The left knee was used as control. No sham operations were performed, since no cartilage changes have been shown to be induced by this procedure. ${ }^{5}$ Postoperatively all animals were kept in pens large enough to allow walking and running. They were killed at regular intervals with an overdose of sodium pentothal, the duration of survival varying from 0.5 to 8 months after surgery.

Preparation of sections. Immediately after killing, both knees were opened and the quality of the articular surface was graded by the Indian ink method. ${ }^{4} \mathrm{~A}$ transverse block of articular cartilage with subchondral bone, approximately $5 \mathrm{~mm}$ thick, was excised from the middle region of the patella and femoral trochlea. Specimens were fixed in formol saline, partly decalcified in nitric acid, embedded in paraffin, and serially sectioned at $5 \mu \mathrm{m}$ normal to the plane of the articular surface. Every tenth section was stained with haematoxylin and eosin or alcian blue. 
The remaining cartilage from each articular surface was removed with a scalpel for biochemical analysis.

Histomorphometry. The sections were examined under the light microscope. The thickness of the noncalcified cartilage was measured with an eyepiece micrometer at regular intervals from the centre of the specimens, the articular margins being avoided. Cells were counted at a magnification $\times 400$ with an integrating eyepiece which consisted of a rectangle 0.225 $\mathrm{mm}$ long and $0.037 \mathrm{~mm}$ wide. The number of nuclei was counted by moving the rectangle perpendicularly from the articular surface until the line of calcification was reached, the length of the rectangle being parallel to the cartilage surface. Counts were made on 4 adjacent lines in the middle region of 5 sections. Increasing the number of sections analysed did not significantly modify the results.

Biochemical analysis. The total glycosaminoglycan content was measured by the fixed charge density (FCD) method. ${ }^{8}$ The wet weight of each specimen was determined after soaking in $0.15 \mathrm{M} \mathrm{NaCl}$. The dry weight was determined by drying to constant weight at $67^{\circ} \mathrm{C}$.

\section{Results}

\section{GROSS CHANGES}

The intact cartilage of the operated joints of dogs killed 2 or more months after surgery was thicker.

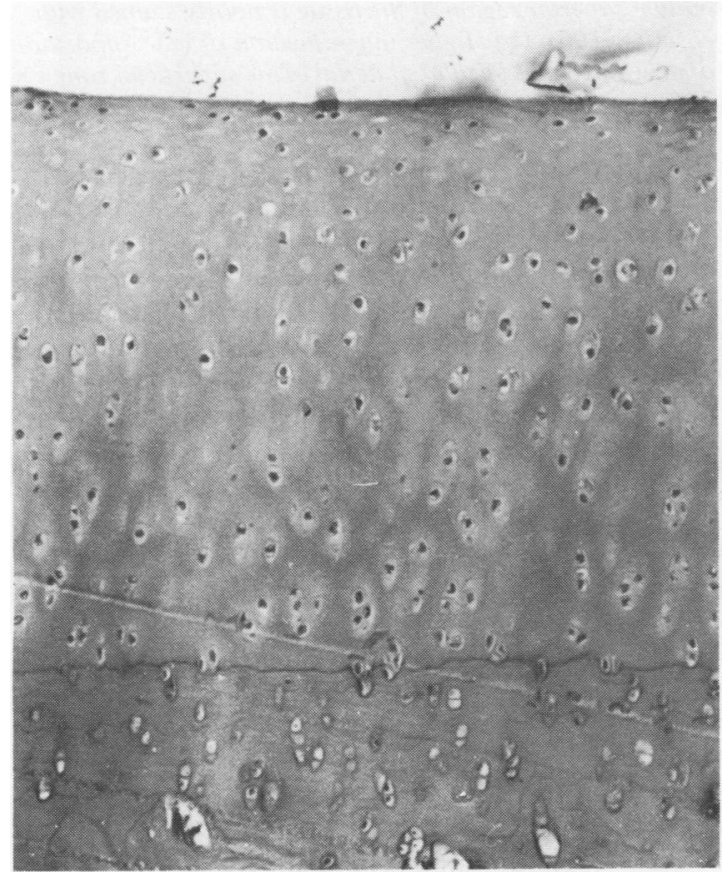

Fig. 1a

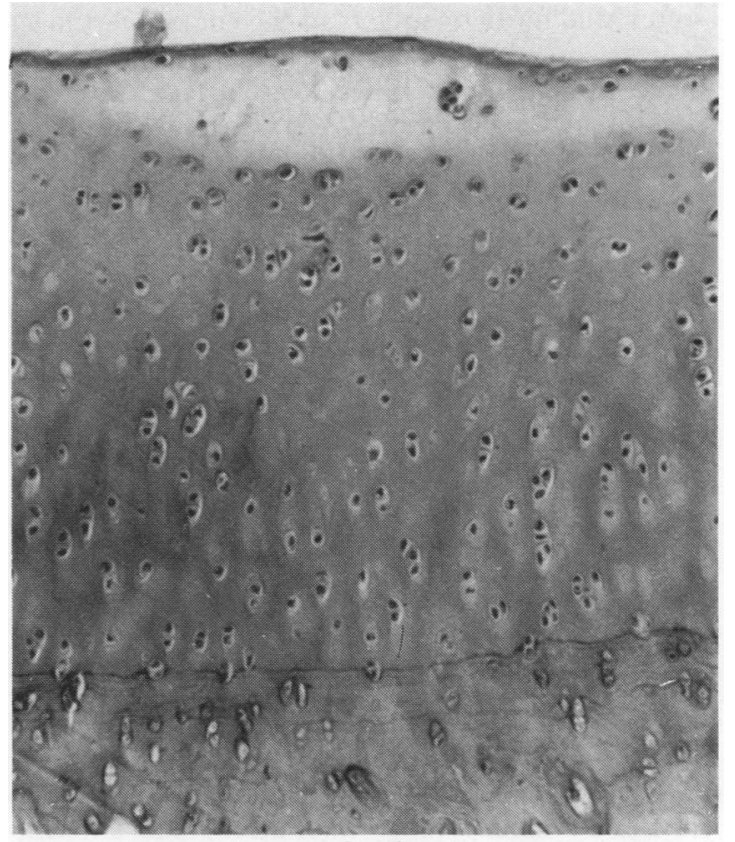

Fig. 1b

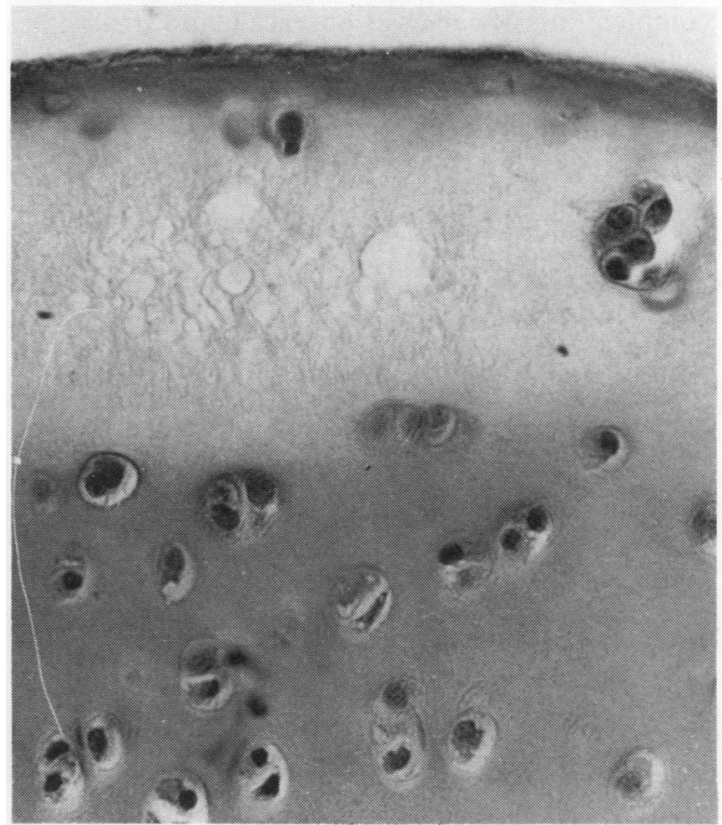

Fig. 1c

Fig. 1 Femoral trochlea cartilage of a dog killed 3 months afier operation (Haematoxylin and eosin). (a) Normal appearance of the control joint $(\times 100)$. (b) Operated joint. Degenerative lesions of the superficial zone and intact articular surface $(\times 100)$. (c) Higher magnification of $(b)$. Clones of 2 or more cells with hypertrophic nuclei around the lacerated matrix $(\times 300)$. 
softer, and more opaque than the control cartilages. In every operated joint the patella and trochlea cartilage showed a generally intact surface, with only occasional small patches of minimal fibrillation demonstrable by Indian ink staining. However, there was a progressive development of prominent osteophytes at the femoral trochlea margins. In contrast, in the medial tibial and femoral condyle cartilage fibrillation was demonstrable as soon as 3 months after surgery and progressed in size and severity, leading to a large zone of deep tissue erosion 7 to 8 months after operation.

HISTOLOGICAL CHANGES

The histological appearance of the cartilage from every control joint was normal. In the operated joints morphological changes were similar in the patella and trochlea cartilage, though more accentuated in the latter. The earliest changes were detectable 2 months after operation. They were mainly degenerative and affected localised areas of the superficial cartilage zone in the central region of the articular surfaces. In these altered areas, the matrix was destroyed, unstained with eosin or alcian blue, and consisted only of a loose fibrillar network; an almost complete loss of cells was observed, and small holes in the altered matrix seemed to indicate the destruction of cells and pericellular lacunae. These areas lay below an intact membrane surface, which usually

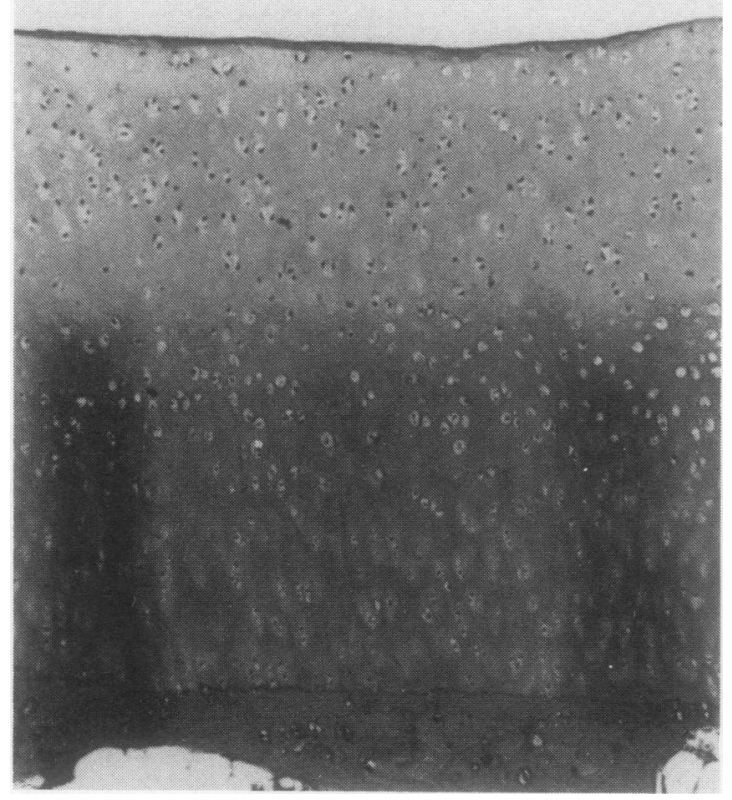

Fig. 2a

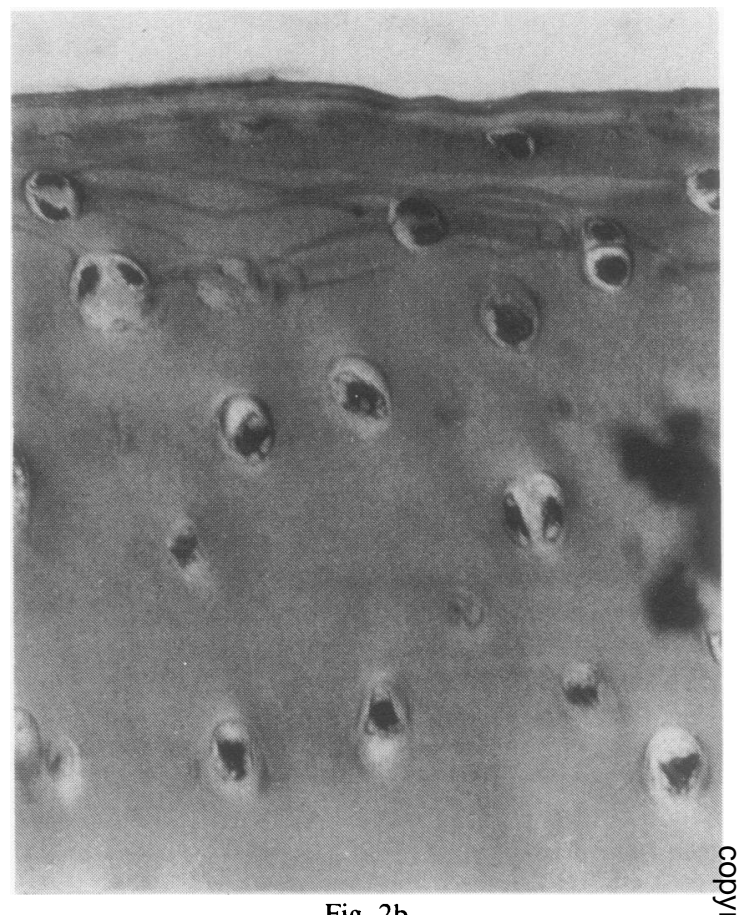

Fig. 2b

Fig. 2 Femoral trochlea cartilage of a dog killed 7 months? after operation. Operated joint (haematoxylin and eosin). (a) Hypertrophic cartilage with a nearly normal appearance. The middle superior region of the tissue is poorly stained with eosin ( $\times 100)$. (b) Higher magnification of (a). Apposition of new tangential fibrillar material in the superficial zone $(\times$ 300).

contained some elongated viable cells, and protruded above the normal contour of the articular surface. These degenerative lesions were associated with regenerative changes, that is, clones of 2 to 5 cells with markedly hypertrophic nuclei in and around the altered matrix, increased thickness of the uncalcified cartilage, and increase matrix staining with alcian blue in the remaining part of the tissue. These superficial lesions were found up to 4 months after operation but never later (Fig. 1).

Surprisingly, the cartilage of the experimental joints of dogs killed 6 or more months after operation had a nearly normal appearance. However, some peculiarities were revealed by careful examination. In the mid-superior part of the cartilage the matrix was poorly stained with eosin but strongly with alcian blue, and enlarged lacunae devoid of sharply defined walls and containing 2 or 3 hypertrophic nuclei were seen. The superficial membrane was markedly thickened by an apposition of tangential fibres, which could be seen running between the hypertrophic cells (Fig. 2). 
MORPHOMETRIC CHANGES

The thickness of the uncalcified cartilage from patella and femoral trochlea was quite similar in the operated and control joints up to 2 months after operation, but thereafter it was greater in the experimental joints. The difference between operated and control joints 3 months after operation was significantly greater than that before 3 months $(p<0.01$; MannWhitney U-test). The mean increase in cartilage thickness from experimental joints obtained 3 to 8 months after operation was nearly $30 \%$.

The variation in cell density with depth from the articular surface from operated and control trochlea cartilage is shown in Fig. 3. A marked decrease in cell number in the 3 most superficial measured zones was evident in each joint obtained more than one month after operation ( $\mathrm{p}<0 \cdot 01$, Wilcoxon's $t$ test). The cell density remained unchanged in the deeper part of the tissue from operated joints. The total cell number was, firstly, reduced due to decreased superficial cell density, and secondly increased by the increased number of zones of measurements and not significantly different from the control side for the whole population (Table 1).

FIXED CHARGE DENSITY AND WATER CONTENT The cartilage from operated joints obtained more than one month after operation had a slightly but significantly higher water content than the control joints $(<0 \cdot 01)$. A similar slight increase in the FCD was found in operated joints when expressed on a dry weight basis, but no significant difference existed between the operated and control joints when the FCD was expressed on a wet weight basis (Table 2).

\section{Discussion}

This work shows that joint laxity produced by section of the anterior cruciate ligament induces 2 major histological changes which precede the onset of cartilage fibrillation.

Hypertrophy of uncalcified cartilage from the
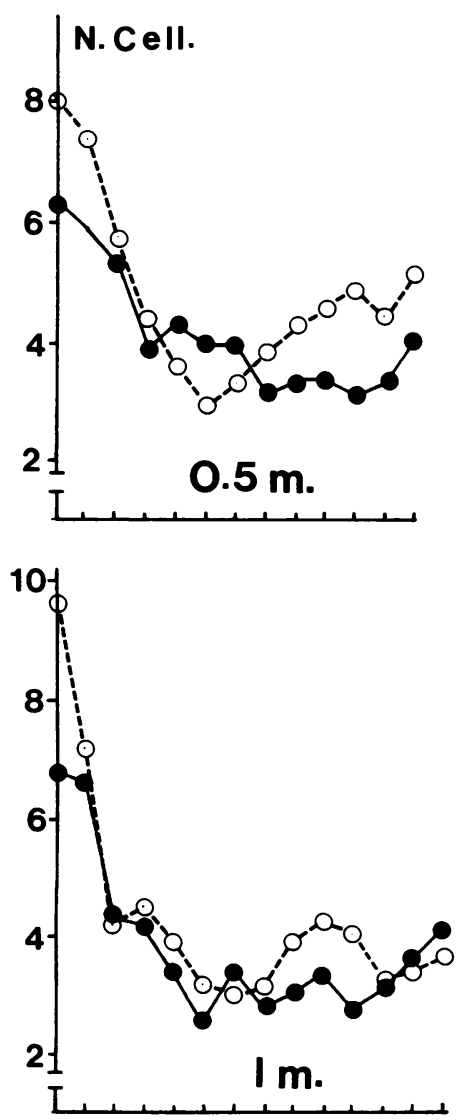
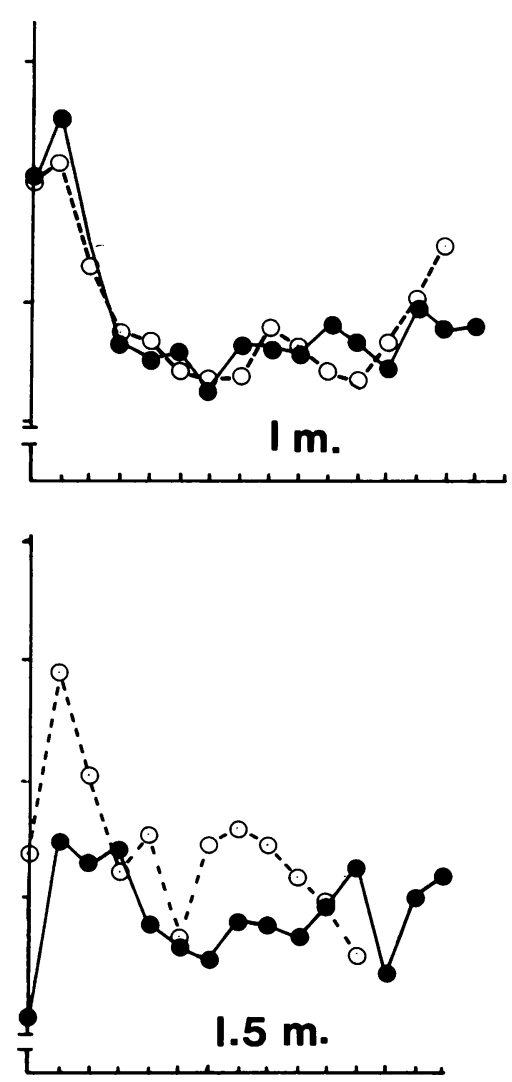

Fig. 3a
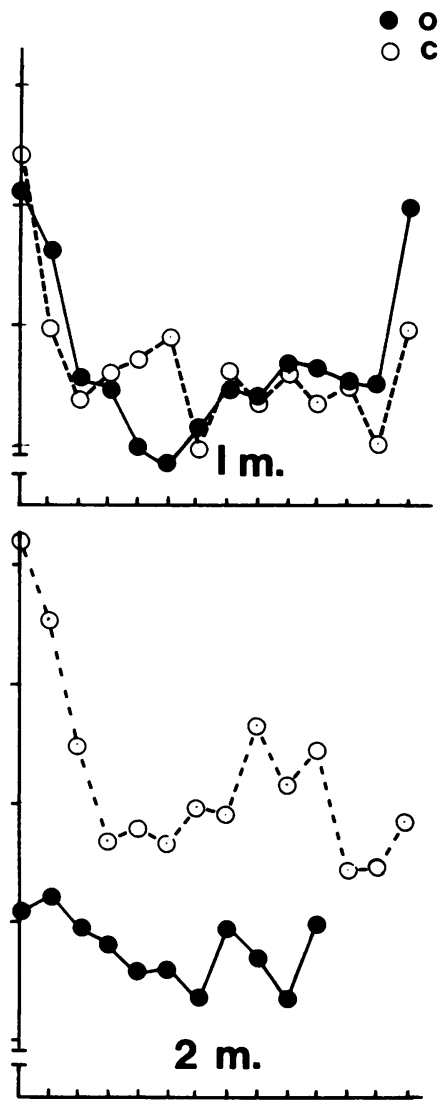

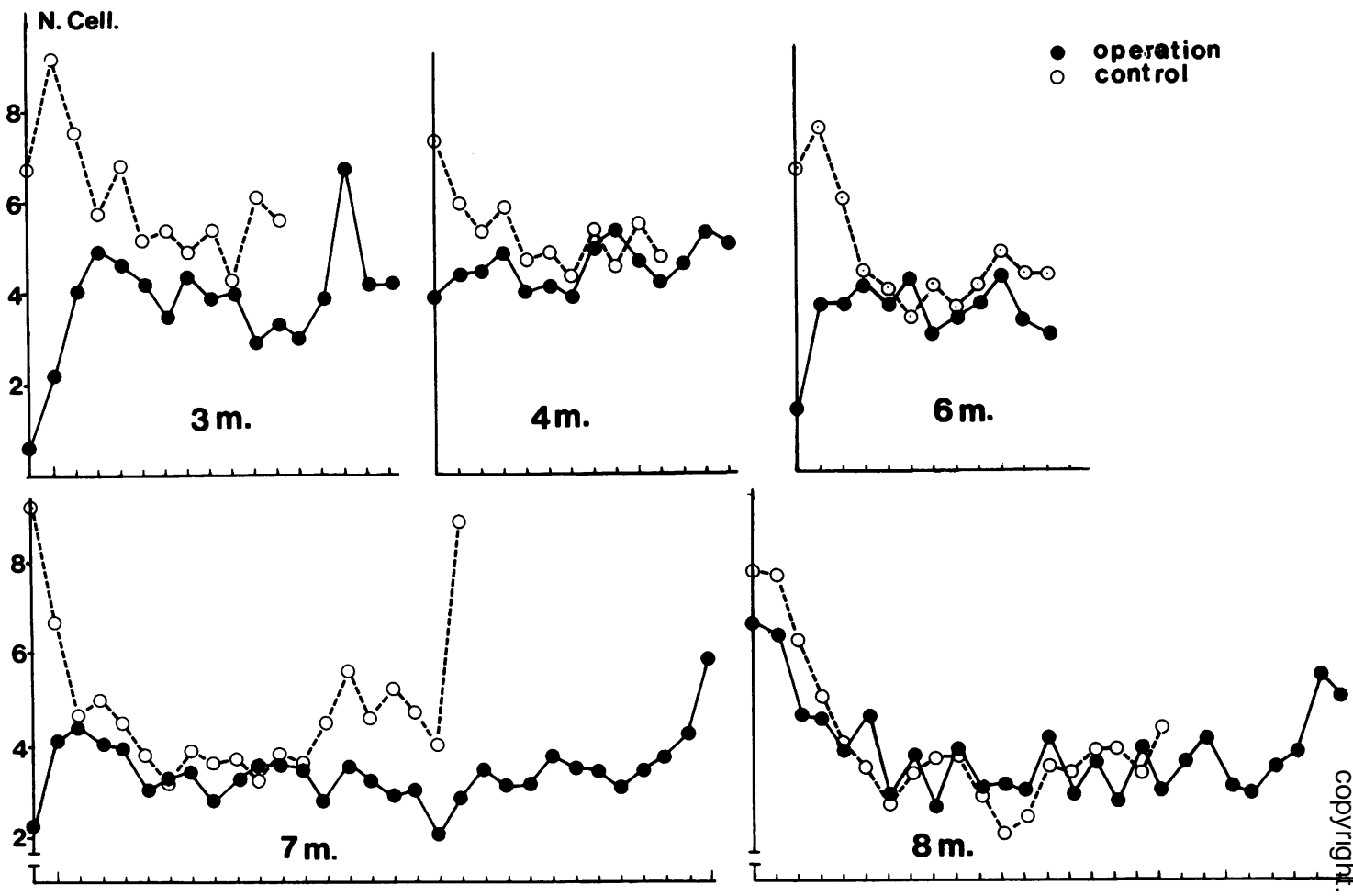

Fig. 3b

Figs. $3 \mathrm{a}$ and b Variations of cell density with distance from the articular surface. Cartilage of femoral trochlea from operated $(O)$ and control $(C)$ joints. (number of nuclei per $8 \cdot 10^{-3} \mathrm{~mm} .^{2}$ Each zone of measurement is $37 \mu \mathrm{m}$ thick. $m=$ Months after operation).

patellofemoral surfaces is the most striking change. The increase in cartilage thickness is clearly shown by comparing dogs killed more than 3 months after operation with those killed sooner and by the magnitude of the difference between the operated and control sides after 3 months.
A greater thickness of the cartilage of experimental joints has previously been suggested and attributed to an increased water content. ${ }^{5}$ Increased hydratation preceding the appearance of fibrillation was in fact found in the present work, as elsewhere, ${ }^{9} 3$. but is probably not the main cause of cartilage hyper-

Table 1 Thickness and total cell number of uncalcified cartilage from patella and femoral trochlea in operated (O) and control (C) joints

\begin{tabular}{|c|c|c|c|c|c|c|c|c|c|c|}
\hline \multirow[t]{2}{*}{ Months after operation } & \multicolumn{2}{|l|}{0.5} & \multicolumn{2}{|l|}{1} & \multicolumn{2}{|l|}{1} & \multicolumn{2}{|l|}{1} & \multicolumn{2}{|l|}{$1 \cdot 5$} \\
\hline & $O$ & $C$ & $O$ & C & $O$ & $C$ & $O$ & C & $O$ & $C$ \\
\hline Patella cartilage thickness (mm) & $0 \cdot 75$ & 0.80 & - & - & 0.73 & $0 \cdot 81$ & 0.96 & $1 \cdot 02$ & - & - \\
\hline Trochlea cartilage thickness (mm) & 0.48 & 0.49 & $0 \cdot 77$ & 0.73 & 0.62 & 0.48 & 0.67 & 0.73 & 0.49 & 0.46 \\
\hline Trohlea total cell number & $53 \cdot 4$ & $62 \cdot 4$ & $61 \cdot 3$ & $57 \cdot 1$ & 49 . & $46 \cdot 6$ & $53 \cdot 8$ & $60 \cdot 9$ & $55 \cdot 7$ & $57 \cdot 5$ \\
\hline
\end{tabular}

\begin{tabular}{|c|c|c|c|c|c|c|c|c|c|c|c|c|}
\hline \multirow[t]{2}{*}{ Months after operation } & \multicolumn{2}{|l|}{2} & \multicolumn{2}{|l|}{3} & \multicolumn{2}{|l|}{4} & \multicolumn{2}{|l|}{5} & \multicolumn{2}{|l|}{7} & \multicolumn{2}{|l|}{8} \\
\hline & $O$ & $C$ & $O$ & $C$ & $O$ & $C$ & $O$ & C & $O$ & C & $O$ & C \\
\hline Patella cartilage thickness (mm) & 0.48 & 0.55 & 0.86 & 0.77 & 0.62 & 0.53 & 0.65 & $0 \cdot 40$ & 0.99 & $0 \cdot 37$ & $0 \cdot 76$ & 0.69 \\
\hline Trochlea cartilage thickness $(\mathrm{mm})$ & $0 \cdot 41$ & 0.48 & 0.60 & 0.41 & 0.51 & 0.42 & 0.42 & 0.40 & $1 \cdot 14$ & $0 \cdot 72$ & $0 \cdot 90$ & 0.62 \\
\hline Trochlea total cell number & $38 \cdot 6$ & $90 \cdot 5$ & $64 \cdot 5$ & $71 \cdot 9$ & $63 \cdot 6$ & $58 \cdot 7$ & $43 \cdot 3$ & $57 \cdot 3$ & $108 \cdot 4$ & $98 \cdot 5$ & $104 \cdot 9$ & $77 \cdot 9$ \\
\hline
\end{tabular}


Table 2 Fixed charge density and water content of patella and femoral trochlea cartilage; comparison between the operated $(O)$ and control $(C)$ joints

\begin{tabular}{|c|c|c|c|c|c|c|c|c|c|c|}
\hline \multirow[t]{2}{*}{ Months after operation } & \multicolumn{2}{|l|}{0.5} & \multicolumn{2}{|l|}{1} & \multicolumn{2}{|l|}{1} & \multicolumn{2}{|l|}{1} & \multicolumn{2}{|l|}{$1 \cdot 5$} \\
\hline & $O$ & $C$ & $O$ & $C$ & $O$ & $C$ & $O$ & $C$ & $O$ & $C$ \\
\hline \multicolumn{11}{|l|}{ Patella } \\
\hline FCD mEq/g wet weight & $0 \cdot 115$ & $0 \cdot 119$ & 0.079 & 0.059 & 0.078 & 0.083 & $0 \cdot 148$ & 0.133 & 0.119 & 0.094 \\
\hline FCD mEq/g dry weight & 0.329 & $0 \cdot 350$ & $0 \cdot 344$ & $0 \cdot 233$ & $0 \cdot 314$ & 0.408 & $0 \cdot 518$ & 0.441 & 0.360 & $0 \cdot 193$ \\
\hline $\mathrm{H}_{2} \mathrm{O} \%$ wet weight & 65 & 66 & $77 \cdot 2$ & $73 \cdot 4$ & $75 \cdot 5$ & $79 \cdot 6$ & $71 \cdot 4$ & 70 & $66 \cdot 9$ & $47 \cdot 6$ \\
\hline \multicolumn{11}{|l|}{ Trochlea } \\
\hline FCD mEq/g wet weight & 0.097 & $0 \cdot 106$ & 0.038 & 0.041 & 0.029 & 0.050 & $0 \cdot 123$ & $0 \cdot 145$ & $0 \cdot 114$ & $0 \cdot 119$ \\
\hline FCD mEq/g dry weight & 0.272 & $0 \cdot 271$ & $0 \cdot 145$ & $0 \cdot 186$ & $0 \cdot 170$ & $0 \cdot 259$ & 0.413 & 0.489 & 0.345 & 0.294 \\
\hline $\mathrm{H}_{2} \mathrm{O} \%$ wet weight & 64.9 & $60 \cdot 6$ & $73 \cdot 8$ & 78 & $82 \cdot 9$ & $80 \cdot 1$ & $70 \cdot 1$ & $69 \cdot 9$ & 66.6 & $58 \cdot 7$ \\
\hline
\end{tabular}

\begin{tabular}{|c|c|c|c|c|c|c|c|c|c|c|c|c|}
\hline \multirow[t]{2}{*}{ Months after operation } & \multicolumn{2}{|l|}{2} & \multicolumn{2}{|l|}{3} & \multicolumn{2}{|l|}{4} & \multicolumn{2}{|l|}{6} & \multicolumn{2}{|l|}{7} & \multicolumn{2}{|l|}{8} \\
\hline & $O$ & $C$ & $O$ & $C$ & $O$ & $C$ & $O$ & $C$ & $O$ & $C$ & $O$ & $C$ \\
\hline \multicolumn{13}{|l|}{ Patella } \\
\hline FCD $\mathrm{mEq} / \mathrm{g}$ wet weight & $0 \cdot 137$ & $0 \cdot 147$ & 0.137 & $0 \cdot 125$ & $0 \cdot 124$ & $0 \cdot 120$ & - & - & - & - & $0 \cdot 155$ & $0 \cdot 120$ \\
\hline FCD mEq/g dry weight & 0.460 & 0.397 & 0.426 & 0.333 & $0 \cdot 398$ & $0 \cdot 318$ & 0.372 & - & 0.492 & 0.411 & - & - \\
\hline $\mathrm{H}_{2} \mathrm{O} \%$ wet weight & $69 \cdot 9$ & $62 \cdot 8$ & $67 \cdot 3$ & $62 \cdot 5$ & $68 \cdot 8$ & $62 \cdot 2$ & - & - & - & - & - & - \\
\hline \multicolumn{13}{|l|}{ Trochlea } \\
\hline FCD mEq/g dry weight & 0.426 & 0.338 & 0.437 & $0 \cdot 240$ & $0 \cdot 264$ & $0 \cdot 276$ & 0.321 & $0 \cdot 214$ & 0.543 & $0 \cdot 288$ & - & - \\
\hline $\mathrm{H}_{2} \mathrm{O} \%$ wet weight & 62 & $61 \cdot 6$ & 69 & $59 \cdot 2$ & $66 \cdot 9$ & $65 \cdot 6$ & - & - & - & - & - & - \\
\hline
\end{tabular}

trophy. The normal or high glycosaminoglycan content found here and in other studies ${ }^{59}$ rather suggests that cartilage hypertrophy is related to an increased synthesis of matrix components. Metabolic studies using the same experimental model of osteoarthritis and showing an increase in proteoglycan and collagen biosynthesis are in close agreement with this view. $^{39 \text { 10-12 }}$

Cell hyperactivity is probably associated with division of middle and deep zone chondrocytes. This is suggested by the normal or high total cell number despite the reduction of the superficial zone cell density and by the normal cell density of the middle and deep zones in spite of the increased thickness of the tissue. Cell division following section of the anterior cruciate ligament is also suggested by histological studies showing clones of 2 or more cells and by metabolic studies showing an increased incorporation of tritiated thymidine. ${ }^{41314}$

Cartilage hypertrophy due to cell division and hyperactivity thus appears to be an initial event preceding the appearance of fibrillation in joint-laxityinduced osteoarthritis. These changes indicate an unexpected remodelling capacity of the tissue in a reactive adaptation to induced abnormal mechanical stresses. The growth of marginal osteophytes can be similarly interpreted and indicates that the reactive process to abnormal stress is not confined to the articular cartilage but affects the joint structures as a whole. It is worth mentioning that hypertrophic changes can be found in nonfibrillated human osteo- arthritic cartilage but only in some cases, so that they do not represent the hallmark of every variety of osteoarthritis. ${ }^{15}$

The changes which we have observed in the superficial cartilage zone are strikingly different from those of the deeper cartilage layers and indicate that a separate analysis of the different tissue layers is advisable for any biochemical or metabolic study. The superficial changes induced by joint laxity are at first degenerative, and the reduction of the superficial cell density is the earliest osteoarthritic lesion detectable at the histological level.

The necrosis of superficial chondrocytes, which has previously been noted in other studies ${ }^{16}{ }^{17}$ precedes and is probably related to the appearance of degenerative alterations of the superficial matrix. These degenerative alterations are unusual in that they do not consist of a fibrillation of the articular surface but are associated with a loss and disruption of the matrix below the intact surface.

A repair process might be another peculiarity of the superficial alterations. Such a repair process is suggested by the observation that the cartilage of dogs killed more than 6 months after operation does not show any degenerative alterations but a marked thickening of the superficial membrane due to apposition of new tangential fibres. This is also suggested by the proliferation of the remaining hypertrophic viable cells, which may be able to migrate in the lacerated matrix and to achieve its repair. Such a possibility of repair might be relevant to the problem 
of chondromalacia patellae, which may undergo spontaneous recovery.

This work was supported by a grant from INSERM, ATP 76-79-108, no. 012. The authors are grateful to Professor H. Bost and Professor J. G. Evreux for technical assistance.

\section{References}

1 Moskowitz $\mathbf{R}$ W. Experimental models of degenerative joint disease. Semin Arthritis Rheum 1972; 1: 95-114.

2 Davis W, Moskowitz $\mathbf{R}$ W. Degenerative joint changes following posterior cruciate ligament section in the rabbit. Clin Orthop 1973; 93: 307-11.

3 Ehrlich M G, Mankin H L, Jones H, et al. Biochemical confirmation of an experimental osteoarthritis model. $J$ Bone Joint Surg 1975; 57A: 392-6.

4 McDevitt C A, Muir H. Gilbertson E. An experimental model of osteoarthritis. Early morphological and biochemical changes. J Bone Joint Surg 1977; 59B: 24-35.

5 McDevitt C A, Muir $\mathrm{H}$. Biochemical changes in the cartilage of the knee in experimental and natural osteoarthritis in the dog. J Bone Joint Surg 1976; 58B: 94-101.

6 Telhag J, Lindberg L. A method for inducing osteoarthritic changes in the rabbit's knees. Clin Orthop 1972; 86: 214-23.

7 Meachim G. Light microscopy of indian ink preparations of fibrillated cartilage. Ann Rheum Dis 1972; 31: 457-9.
8 Maroudas A, Thomas H. A simple physicochemical micromethod for determining fixed anionic groups in connective tissue. Biochim Biophys Acta 1970; 215: 214-21.

9 Palmoski M J, Coyler R A, Brandt K D. Marked suppression by salicylate of the augmented proteoglycan synthesis in osteoarthritic cartilage. Arthritis Rheum 1980; 23: 83-91.

10 Gold E W, Anderson L B, Miller C W, Schwartx E R. Effect of salicylate on the surgical inducement of joint degeneration in rabbit knees. J Bone Joint Surg 1976; 52A: 1012-5.

11 Eyre D R, McDevitt C A, Billingham M E J, Muir $H$. Biosynthesis of collagen and other matrix proteins by articular cartilage in experimental osteoarthritis. Biochem $J$ 1980; 183: 823-37.

12 Floman $\mathrm{Y}$, Eyre $\mathrm{D} \mathbf{R}$, Glimcher $\mathbf{M}$. Induction of osteoarthritis in rabbit knee joint: biochemical studies on the articular cartilage. Clin Orthop 1980; 147: 278-86.

13 Schartz E R. Metabolic response during early stage of surgically induced osteoarthritis in mature beagles. $J$ Rheumatol 1980; 7: 788-800.

14 Telhag $H$. Mitosis of chondrocytes in experimental osteoarthritis in rabbits. Clin Orthop 1972; 86: 224-9.

15 Vignon E, Arlot M. Macroscopically normal cartilage from the human osteoarthritic femoral head. $J$ Rheumatol in press.

16 Pond M J, Nuki G. Experimentally induced osteoarthritis in the dog. Ann Rheum Dis 1973; 32: 387-8.

17 Lufti A M. Morphological changes in the articular cartilage after meniscectomy. J Bone Joint Surg 1975; 57B: 525-8. 